\title{
L'accueil du piéton dans les espaces publics du centre-ville de Marseille : discours, pratiques, actions
}

\author{
Pedestrians in public spaces of Marseille's city centre: views, practices, actions
}

\author{
Brigitte Bertoncello · Frédérique Hernandez · Angelo Bertoni
}

Reçu le $1^{\text {er }}$ décembre 2012 ; accepté le 28 octobre 2013

(C) IFSTTAR et Éditions NecPlus 2013

Résumé Marseille est depuis plus de dix ans une ville en chantier dans laquelle sont conduits des travaux de requalification du centre-ville, incluant une intervention sur le bâti mais aussi sur la mobilité avec la volonté affichée de réguler la circulation automobile. Un des enjeux de cette requalification urbaine est d'attirer une population nouvelle tant en termes de futurs résidents que de futurs visiteurs. La ville tente d'améliorer la qualité et le confort du centre-ville : la prise en compte du piéton devient un élément incontournable de la qualité urbaine et une attention particulière est consacrée aux espaces publics. Cet article montre, dans un premier temps, que les programmations réalisées et en cours, tant en matière de requalification d'espaces publics qu'en matière de réorganisation des déplacements, sont guidées par un objectif de redynamisation du centre-ville et

Les auteurs tiennent à remercier Patricia Antalovsky (Pôle ressources et données urbaines) et Vincent Tinet (Pôle développement durable environnement et mobilités) de l'Agence d'urbanisme de l'agglomération marseillaise (Agam) pour leur disponibilité et leur aide dans l'accès aux documents et données chiffrées utiles à la réalisation de cet article.

Frédérique Hernandez $(\bowtie)$

Aix Marseille Université

Institut d'Urbanisme et d'Aménagement Régional,

CIRTA/LIEU EA 889

3, avenue Robert Schuman

F-13628 Aix-en-Provence cedex 01, France

e-mail : frederique.hernandez@univ-amu.fr

Brigitte Bertoncello $(\bowtie)$

Aix Marseille Université

Institut d'Urbanisme et d'Aménagement Régional,

CIRTA/LIEU EA 889

3, avenue Robert Schuman

F-13628 Aix-en-Provence cedex 01, France

e-mail : brigitte.bertoncello@univ-amu.fr

Angelo Bertoni $(\bowtie)$

Aix Marseille Université

Institut d'Urbanisme et d'Aménagement Régional,

UMR TELEMME 7303 (AMU, CNRS)

3, avenue Robert Schuman,

F-13628 Aix-en-Provence cedex 01, France

e-mail : angelo.bertoni@univ-amu.fr d'amélioration de son image. Cette redynamisation est avant tout d'ordre économique et particulièrement centrée sur le développement commercial. Aussi, la ville a-t-elle tendance à se focaliser sur les populations actives et consommatrices au détriment des populations résidentes. Dans un second temps, l'article met en évidence que la marche est envisagée comme un flux et non comme un mode de vie. Une marche dynamique, utile, qui laisse peu de place à la flânerie est privilégiée. L'étude du Cours Belsunce, espace de l'hypercentre marseillais, montre à quel point un espace public conçu pour la promenade et la pause, voit sa fonction initiale détournée au profit du transit.

Mots clés piéton - espaces publics - marche dynamique · hypercentre $\cdot$ requalification · Marseille $\cdot$ Cours Belsunce.

Abstract For over ten years Marseille has been a city under construction. Rehabilitation works have being carried out on the city centre, including building intervention and improved mobility with a focus on traffic regulation. The challenges of this urban regeneration is to attract a new population of future residents and future visitors. The city seeks to improve the quality and comfort of the city centre: the inclusion of the pedestrian dimension becomes an essential element of urban quality and special attention is given to public spaces. This article shows, firstly, that programmes on public spaces rehabilitation and on mobility reorganization - completed or in progress - are aimed at revitalizing the city centre and improving its image. This revitalization is primarily economic and particularly focused on business development. Furthermore, the city of Marseille tends to focus on the working population and consumers at the detriment of residents. Subsequently, the article shows that walking is considered as a flow and not as a lifestyle. We refer to a dynamic and useful walking, with little opportunity for flanerie. The case of Cours Belsunce, in Marseille city core, shows how a public space designed for walking and staying, sees its initial function diverted in favour of transit.

Keywords Pedestrian · Public spaces · Dynamic walking . City core $\cdot$ Marseille $\cdot$ Cours Belsunce 


\section{Introduction}

Le positionnement des villes et leur dynamisme semblent passer par l'image de leur centre-ville. À Marseille, ce centre resté grandement populaire et caractérisé par la présence d'une population d'origine étrangère impliquée dans une activité commerciale aux produits bon marché, ne correspond pas aux attendus du gouvernement local ni au standing habituel des métropoles.

Malgré plus de quarante ans de politiques de transformation de ce centre avec la ferme intention d'attirer de nouvelles populations, la situation reste insatisfaisante. Certes des immeubles ont été réhabilités, des espaces publics réaménagés, de nouvelles infrastructures construites mais le changement d'image attendu n'est pas au rendezvous. Pour certains auteurs ce résultat mitigé s'apparente à une forme de résistance des populations en place face aux programmations urbanistiques [1]. Il pourrait également être lié au fait que les interventions ont été fortement centrées sur le logement, le bâti et sa réhabilitation au détriment de l'habitat, du cadre de vie et des espaces publics [2]. Ces derniers ont été abordés ponctuellement ou de manière circonscrite, ils n'ont pas été placés au cœur d'une intervention en termes de composition urbaine ${ }^{1}$. Ils sont aujourd'hui pratiqués par une société en recomposition, faite d'habitants issus d'une migration internationale à petits revenus et avec une faible qualification professionnelle et de citadins venant d'autres quartiers de Marseille, mais aussi de nouveaux résidents, de cadres et d'employés attirés par ce quartier en transformation. Cette société plurielle croise, dans les espaces publics récemment aménagés, les touristes venus visiter le centre-ville ainsi que les consommateurs de la région attirés par un ensemble de commerces alliant produits bon marché et enseignes franchisées.

Marseille est aujourd'hui une ville en chantier dans laquelle sont conduits des travaux de requalification du centre-ville - incluant une intervention sur le bâti mais aussi sur la mobilité - avec la volonté affichée de réguler la circulation automobile. La récente semi-piétonisation du Vieux-Port s'accompagne d'une redistribution des déplacements en cœur de ville ; l'opération vise à réduire la place de la voiture dans l'hypercentre au profit des modes doux, particulièrement la marche. Dans ce contexte les espaces publics méritent une attention particulière ; ils sont en effet au croisement de la redynamisation du centre et de l'apaisement de la circulation. Leur devenir s'inscrit dans une dynamique globale de changement d'image destinée à rendre le centre attractif.

\footnotetext{
${ }^{1}$ La composition urbaine correspond à une vision d'ensemble en vue d'articuler différents éléments dans un tout soumis au principe d'unité ; aucune partie de ce tout ne prétend suffire à elle-même.
}

\section{Problématique}

Un des enjeux de cette requalification urbaine est d'attirer une population nouvelle tant en termes de futurs résidents que de futurs visiteurs (touristes, chalands...). Pour ce faire la municipalité tente d'améliorer la qualité et le confort du centre-ville. Et à l'instar d'autres agglomérations, Marseille considère la prise en compte du piéton comme un élément incontournable de la qualité urbaine et accorde ainsi une attention particulière à ses espaces publics. Au regard des efforts entrepris par la ville, depuis plusieurs années pour d'une part, séduire une nouvelle population, et d'autre part euphémiser la présence d'une population dont l'identité va à l'encontre de l'image recherchée, il est légitime de se demander si les attentes des actuels résidents sont dans un même temps satisfaites $[3,4,5]$. Sur ce point, la politique de requalification du centre semble avoir été menée à contretemps : pas pour les habitants en place, mais pour une population différente, fantasmée, que les édiles souhaiteraient voir arriver. Quelle incidence cette quête d'attractivité a-t-elle sur les aménagements des espaces publics et particulièrement sur ceux qui sont destinés aux piétons ? Nous faisons l'hypothèse que la politique d'aménagement des espaces publics favorise une " marche dynamique » au détriment d'une «marche contemplative ». Cette «marche dynamique » ne répond pas aux besoins et aux enjeux de la mobilité piétonne des résidents du centre-ville à faibles revenus et ne possédant pas d'automobile.

Dans un premier temps, cet article montre que les programmations réalisées et en cours (tant en matière de requalification d'espace public qu'en matière de réorganisation des déplacements) sont effectivement guidées par un objectif de redynamisation du centre-ville et d'amélioration de son image. Cette redynamisation, avant tout d'ordre économique, est particulièrement centrée sur le développement commercial. Dans un second temps, nous mettons en évidence que la marche est envisagée comme un flux et non comme un mode de vie. Et tant bien même la nature de l'espace public se prêterait à la promenade et la pause - comme le suggèrent certaines figures d'espaces publics (cours, mail...) -, sa fonction initiale est détournée au profit d'un espace de transit.

Le développement de cette thèse s'appuie sur différentes méthodes : analyses de documents d'urbanisme, exploitation des enquêtes-déplacements, observations des pratiques. Pour mettre à jour les stratégies qui sous-tendent le contenu des projets de requalification et d'aménagement de l'espace public - et véritablement comprendre la place du piéton dans le dispositif -, nous avons analysé des documents d'urbanisme censés cadrer les interventions envisagées dans le centre-ville de Marseille. Ces documents ont été rédigés 
entre le début des années 1990 (période d'émergence d'une première réflexion d'ensemble sur le centre-ville) et aujourd'hui : le Projet Centre-ville (1995-2001) ; le Schéma directeur des espaces publics du centre-ville de Marseille (2002) ; les deux Plans de déplacements urbains (PDU) de 2000 et 2006 et l'Opération grand centre-ville (2011-2021). Une grille de lecture a été élaborée pour appréhender la place accordée aux espaces publics, la façon dont il est fait référence aux utilisateurs (existants ou potentiels, résidents ou venant d'autres quartiers et d'autres villes, leurs pratiques), les objectifs en matière de développement de la marche (par rapport aux autres modes de déplacements, les outils envisagés pour la favoriser) et, enfin, comment le piéton est évoqué.

Dans les enquêtes ménages-déplacements (1997, 2009), ont été exploitées les données concernant la mobilité des résidents du centre-ville et la part de la marche dans les déplacements liés à cet espace. Des recherches antérieures sur la population de l'hypercentre ont été mobilisées afin de mieux comprendre leur rapport à l'espace public et leur situation en termes de déplacements.

Enfin, ces analyses ont été complétées par des observations sur le terrain, en centre-ville, avec une attention particulière pour un secteur de l'hypercentre : le Cours Belsunce. Il s'est agi d'analyser, d'une part l'organisation spatiale de cet espace public réaménagé suite à l'introduction du tramway et d'autre part les pratiques de ses usagers.

Notre posture de recherche ne consiste pas à aborder le piéton comme une catégorie de la mobilité, mais plutôt comme un acteur porteur d'une pratique : la marche. Les espaces publics sont ainsi appréhendés comme le support de cette pratique.

Cet article comprend un état de l'intérêt renouvelé pour le piéton et la marche en ville, puis un examen de la place faite au piéton à travers une analyse des objectifs et des stratégies qui sous-tendent différents documents d'urbanisme marseillais produits depuis une vingtaine d'années. Est ensuite étudié le cas du Cours Belsunce, espace de l'hypercentre marseillais.

\section{La mobilité piétonne : un enjeu aux multiples échelles entre vitrine et vécu quotidien}

\section{Le renouveau de la marche en ville réinterroge les espaces publics et leur requalification}

Dans les discours sur l'intervention urbaine, le piéton fait l'objet de toutes les attentions. Le développement de la pratique de la marche devient un objectif à atteindre au nom du développement durable et de la qualité urbaine. . mais quelle est la réalité opérationnelle de cet engouement? N'y aurait-il pas une instrumentalisation du piéton au nom de la requalification du cadre de vie oubliant la pratique « libre », première, des espaces publics ?

À partir des années 1950, les théories urbanistiques redécouvrent le rôle du piéton et de la marche en ville en réaction à l'essor de la planification urbaine des années de l'après-guerre. Le monde anglo-saxon réagit en premier : les ténors de l'Urban Design, tels que Kevin Lynch et Gordon Cullen, prônent alors une vision urbaine renouvelée par la prise en compte des pratiques piétonnes, notamment dans les quartiers centraux, en contraste avec l'introduction d'infrastructures autoroutières souvent projetées à cette époque (le cas de Marseille, par ex.). Le piéton est alors synonyme d'animation et d'espaces urbains à taille humaine [6,7]. En Europe, la crise pétrolière des années 1970 fait redécouvrir la marche active comme mode alternatif à l'automobile. Quelques initiatives voient le jour, mais restent liées à des contextes locaux, sans que cela se traduise durablement dans des stratégies d'aménagement. Plus tard, dans les années 1990, le mouvement du New Urbanism de matrice étasunienne, met le piéton au centre des aménagements à l'échelle du quartier et fait de la « marchabilité » une caractéristique essentielle du fait urbain et suburbain [8]. L'espace public s'affirme comme lieu de déambulation et de rencontres, indissociable de caractères d'aménité. À la fin du XX $\mathrm{XX}^{\mathrm{e}}$ siècle, l'intérêt croissant pour le cadre de vie et les éléments qui en déterminent l'urbanité (logement, travail, éducation, culture) placent l'accessibilité piétonne comme un droit des habitants [10].

Si la marche est considérée comme la couche première qui structure une politique de mobilité, les espaces publics en deviennent le corollaire incontournable et constituent l'élément clé dans l'articulation entre réseaux de transport et opérations urbaines [9]. L’opportunité de séjourner dans des espaces publics adaptés et de qualité devient un levier important pour la pratique de la marche.

Certaines villes ont répondu, dans les dernières années, à une carence des dispositifs liés à la marche urbaine en mettant en cohérence des politiques sectorielles [11] : la marche est pensée à l'échelle de l'agglomération, dans la perspective de réaliser la ville des courtes distances. La continuité des aménagements entre centres et périphéries devient indispensable.

Laurent Turcot et Sonia Lavadinho contribuent depuis les années 2000 à remobiliser l'attention de la communauté scientifique sur le piéton en ville. Leurs productions orientées vers la promenade ou la marche urbaine montrent comment ces modes sont devenus des éléments centraux dans les discours sur la ville $[12,13]$. Le piéton touriste-marcheur devient un des principaux destinataires de la promotion urbaine. La vie culturelle de nombreuses villes occidentales repose ainsi dans ses manifestations les plus médiatisées sur l'appréhension de la ville par le piéton (les Nuits Blanches à 
Paris, la Nuit des Musées mis en place dans de nombreuses villes, ou encore le Museum Mile à Londres, etc.).

Si le piéton reste une figure neutre, à l'identité rarement précisée, plusieurs types de marche sont distingués par les auteurs : certains parlent de "marche joyeuse ", quand elle s'épanouit dans la rue marchande ou habitante [14], d'autres de " marche utilitaire », si elle emprunte des lieux-mouvements dévolus aux transports publics [15], d'autres encore de " marche découragée », s'agissant de terrains vagues où l'urbanité reste faible [16].

Malgré la diversité des cultures et des modes de vie urbains, certains aménagements pionniers des espaces piétons mis en œuvre dans les pays du Nord de l'Europe, se généralisent aujourd'hui. Ils sont caractérisés par des trottoirs élargis, bordés d'un décor choisi pour ses qualités esthétiques et l'image qu'il confère à la ville (lampadaires stylisés, bacs à fleurs ou bornes garde-fous...), par l'élimination d'obstacles et par des surfaces aplanies, par des revêtements soignés. À Grenoble, comme à Genève, Munich ou Lyon, le piéton marche dans un environnement quasi-lisse et souvent dégagé, où l'espace à dominante minérale est agrémenté de végétaux en tous genres.

Ces environnements urbains destinés aux piétons et aux caractéristiques de plus en plus standardisées ont fait l'objet d'un travail récent, mené par l'équipe de recherche du Cresson (Grenoble). Une analyse originale y est proposée, construite à partir de l'étude des ambiances urbaines. L'équipe a montré à quel point l'aménagement matériel apparaît moins déterminant que les qualités d'ambiance dans le choix d'un trajet et le plaisir ou le déplaisir de marcher dans telle rue ou dans tel quartier. Remettant en cause le déterminisme strict entre l'espace matériel et l'activité piétonne, l'enquête sur deux quartiers de Grenoble a montré l'intérêt de penser l'espace du déplacement à pied en termes de potentiel d'action, un espace pouvant être parcouru et fréquenté de diverses manières, laissant des possibilités de choix et de variations [17]. Ces résultats peuvent néanmoins être nuancés par les analyses de Sylvie Miaux portant sur la relation entre la manière d'envisager la marche et la perception de l'environnement urbain par le piéton. Cette auteure constate l'influence du statut socioéconomique dans la manière de concevoir la marche. Les populations sans ressources et sans voiture, vivent majoritairement la marche comme une contrainte ou une nécessité, les populations à revenus moyens et supérieurs, possédant une ou plusieurs voitures, marchent par engagement. Elle montre que ces différentes manières de vivre la marche influencent différemment la relation à l'environnement urbain et à autrui. Le piéton qui marche par engagement porte une attention particulière à l'ambiance et aux caractéristiques esthétiques de l'environnement urbain, alors que le piéton qui marche par contrainte et par nécessité se préoccupe principalement de sa sécurité (routière et publique) et attache moins d'importance à la qualité de l'environnement urbain [18].

$\mathrm{Au}$ final, les piétons sont mus par des motifs divers et appréhendent différemment la pratique de la marche et leur environnement. Comment les concepteurs et les gestionnaires des espaces publics travaillent-ils à l'équilibre des fonctions et intègrent-ils identités et attendus des multiples usagers?

\section{À Marseille, une mobilité piétonne essentielle à la vie quotidienne d'un centre majoritairement populaire}

En 1997, la municipalité de Marseille s'aperçoit que le nombre de déplacements piétons dans son centre a diminué de $40 \%$ par rapport à 1976 . Cette baisse du nombre de déplacements piétons est en partie due à une baisse démographique dans le centre et pour partie à un report sur les modes motorisés (transports collectifs et, principalement, la voiture). Il est alors important pour la ville tant d'augmenter la démographie de son centre-ville (Projet centre-ville) que de faire progresser la part des déplacements piétons dans son centre (Plan de déplacements urbains - PDU). Depuis, le nombre de résidents dans le centre a augmenté ainsi que la part modale de la marche. La dernière enquête ménages-déplacements (2009) révèle que 3210000 déplacements ont lieu chaque jour dans Marseille (flux liés au territoire), dont 994000 concernent le centre-ville. Ce dernier chiffre est en augmentation de $23 \%$ par rapport à 1997. Cette augmentation est principalement due à l'augmentation démographique de la commune (qui gagne 82000 habitants, dont 30000 pour le centre-ville). Concernant la part modale des flux liés au centre-ville ${ }^{2}$, $71 \%$ des déplacements se font par la marche en mode principal, $14 \%$ des déplacements se réalisent en voiture, $12 \%$ en transports collectifs. Entre 1997 et 2009, la part modale de la marche a augmenté dans le centre-ville (passant de $65 \%$ à $71 \%$ ) et la part de la voiture a baissé (passant de $22 \%$ à $14 \%^{3}$ ). Deux déplacements à pieds sur trois dans l'hypercentre sont le fait des résidents de ce secteur ${ }^{4}$. Cette donnée montre l'enjeu que représente une réflexion sur la mobilité piétonne à l'échelle du résident.

\footnotetext{
${ }^{2}$ Il s'agit ici de comptabiliser pour un périmètre les déplacements entrant, sortant ou interne à celui-ci, sans tenir compte du lieu de résidence des personnes s'y déplaçant.

${ }^{3}$ Notons qu'il s'agit ici des «parts modales » et non des distances parcourues par chaque mode. La différence entre les deux indicateurs n'est pas anodine.

${ }^{4}$ Donnée issue de l'enquête ménages-déplacements 1988, présentée dans : Ville de Marseille (DGST, Direction de la voie publique, division circulation signalisation), Ocotram : Plan de déplacements, diagnostic, décembre 1990.
} 
Les habitants du centre-ville (comprenant les arrondissements du $1^{\mathrm{er}}$ au $7^{\mathrm{e}}$ ) réalisent en moyenne 4,04 déplacements par jour de semaine ; ils sont les habitants les plus mobiles de la commune. Pour $55 \%$ de leurs déplacements ils ont recours à la marche, pour $25 \%$ à la voiture et pour $13,8 \%$ aux transports collectifs. Comparés à la mobilité de l'ensemble des habitants de la commune de Marseille, les résidents du centre-ville ont plus souvent recours à la marche, moins souvent recours à la voiture et quasiment autant recours aux transports en commun. Soulignons, que $43 \%$ des ménages du centre-ville sont non-motorisés.

Les résidents de l'hypercentre (comprenant les arrondissements du $1^{\mathrm{er}}$ au $4^{\mathrm{e}}$ ) réalisent en moyenne 4,01 déplacements par jour. Pour $61 \%$ de leurs déplacements ils ont recours à la marche comme mode principal... La part de ménages non-motorisés s'élève à $60 \%$ dans les deux premiers arrondissements de l'hypercentre. De fait, une part importante de la population de l'hypercentre apparaît ainsi particulièrement captive de la marche, des deux-roues et des transports collectifs. Cette donnée est à corréler au faible niveau de ressources d'une grande partie des ménages résidant dans l'hypercentre ${ }^{5}$. La non-possession de la voiture ne s'inscrit pas dans le registre d'un choix assumé, mais semble relever de la contrainte. Pour une grande part de la population paupérisée résidente, le principal mode de déplacement reste la marche (l'utilisation des transports en commun ayant un coût). Si le terme de «dépendance automobile » caractérise la situation des habitants des zones périurbaines, peut-on parler de " dépendance piétonne » ou de "captifs de la marche » pour les habitants des arrondissements hypercentraux de Marseille ? Ces constats accentuent l'intérêt de notre recherche sur la façon dont les aménagements pour les piétons répondent aux besoins des résidents.

\section{La redynamisation commerciale, un objectif qui guide la façon dont les documents stratégiques d'aménagement envisagent le piéton}

À l'instar d'autres métropoles françaises, la ville de Marseille a, le plus souvent en collaboration avec l'Agence d'urbanisme de l'agglomération marseillaise (Agam), d'une

\footnotetext{
${ }^{5}$ Quartier d'accueil de différentes vagues migratoires, l'hypercentre est en effet caractérisé par le faible niveau de qualification des demandeurs d'emploi, des conditions d'habitat inadaptées aux familles nombreuses, du logement social insuffisant (forte proportion de logement social de fait), une dépendance importante aux prestations sociales, de nombreux ménages fiscaux sous le seuil théorique de pauvreté, un bâti dégradé et insalubre.
}

part, mis en place les dispositifs lancés au niveau national (OPAH, PRI, ZPPAUP, PDU, PRI, etc.) et, d'autre part, élaboré de nombreux documents d'urbanisme servant de référence aux interventions dans le centre-ville. Ces différents documents à travers leur(s) regard(s) sur les espaces publics, abordent le piéton, de manière plus ou moins directe, plus ou moins fine.

Le Projet centre-ville (1995-2001) correspond à la première initiative municipale tendant à privilégier un programme d'ensemble sur le centre-ville, avec un élu de référence, Claude Valette ( $1^{\mathrm{er}}$ adjoint à l'urbanisme), fortement impliqué dans «la dynamique de reconquête » d'un territoire considéré comme défaillant.

Une réflexion nouvelle en matière d'organisation des déplacements est engagée à partir de 1995 par la Direction générale des services technique de la ville de Marseille. Ces réflexions donnent lieu à divers diagnostics. Un premier PDU est adopté en novembre 2000, il a été élaboré par la Ville, alors autorité organisatrice des transports urbains, et s'inscrit dans le cadre de la Loi sur l'air et l'utilisation rationnelle de l'énergie (Laure) ${ }^{6}$.

Deux ans après son approbation, un Schéma directeur des espaces publics (SDEP) du centre-ville de Marseille est produit par les services de la ville, mais il ne pourra être appliqué. En effet, la gestion des espaces publics est dorénavant associée à la voirie, une fonction entre-temps prise en charge par la communauté urbaine Marseille-Provence-Métropole (MPM) ${ }^{7}$ qui ne s'est pas saisie officiellement du document existant. Ce document reste toutefois pertinent : il est le premier du genre (et pour l'instant l'unique).

L'Opération grand centre-ville est la toute dernière mobilisation sur un centre-ville élargi ${ }^{8}$, à resituer dans le contexte de l'obtention du label Capitale européenne de la culture en 2013 pour Marseille-Provence. L'objectif de faire du centre-ville un « cœur emblématique de la Cité » s'apparente aux ambitions de l'émergence «d'un cœur métropolitain ». La requalification des espaces publics y occupe une place de choix.

\footnotetext{
${ }^{6}$ En 2006, un second PDU est adopté, inscrit dans le cadre de la loi Solidarité et renouvellement urbain (SRU). Il est conduit par la communauté urbaine Marseille-Provence-Métropole (MPM). Une troisième mouture vient d'être approuvée.

${ }^{7}$ La communauté urbaine MPM est créée par arrêté préfectoral du 7 juillet 2000

${ }^{8}$ Dans la continuité de sa politique de requalification du centre-ville, la Ville de Marseille (par délibération du 9 février 2009) a approuvé un engagement renforcé pour le centre-ville. Dans un même temps, la communauté urbaine MPM (par délibération du 19 février 2009) a confirmé sa volonté de participer à la requalification envisagée qui s'inscrit dans les grands chantiers programmés pour transformer Marseille en métropole méditerranéenne.
} 
L'examen d'un corpus de documents ${ }^{9}$ met en exergue la façon dont les espaces publics et les piétons sont appréhendés. Les paragraphes suivants participent à montrer l'importance accordée à certaines catégories de piétons.

Dans le Projet centre-ville, il est précisé que les actions engagées «sont indissociables de la nécessité de "faire revenir plus de consommateurs" et de mieux satisfaire les besoins des populations résidentes actuelles et futures dans le cadre des projets de réhabilitation qui sont en cours $»^{10}$. Pour ce faire, la Ville a engagé un partenariat avec la Chambre de commerce et de l'industrie (CCI) et l'Association des commerçants plein-centre afin de développer une action pluriannuelle de revitalisation du commerce et de l'artisanat dans le centre-ville. L'ensemble fait l'objet d'une convention Fisac (Fonds d'intervention pour la sauvegarde de l'activité commerciale et artisanale) signée en octobre 1998 et portant sur une période triennale (jusqu'en 2001). C'est en lien avec une meilleure « connaissance des besoins de la clientèle » que pourront être atteints deux objectifs : "Mettre en place une dynamique commerciale du Centre-Ville » et «Valoriser les forces commerciales » en présence. Il s'agit alors de «Favoriser l'accès, le stationnement en Centre-Ville et le déplacement des piétons dans l'Hypercentre » et d' « Améliorer l'environnement du consommateur ». Ces deux derniers objectifs permettent de saisir les enjeux économiques sous-jacents aux programmations urbanistiques. Derrière la requalification du centre, c'est bien la redynamisation du commerce qui est visée. Dans la deuxième phase du projet initialement envisagée (2002-2007), il est question de valoriser « l'achat plaisir » à travers des actions sur l'espace public et la mise en place de programmes d'actions structurants sur les quartiers qui jouxtent l'hypercentre.

Le PDU de la Ville (2000) s'inscrit dans les objectifs du « Schéma de cohérence Marseille 2015 » : tendre vers « une ville qui se développe et qui rayonne » et faire de Marseille « une ville accueillante et agréable à vivre ». Le PDU se positionne dans le registre du fonctionnement urbain, en soulignant que cet aspect « est une condition essentielle au développement économique du projet urbain de la ville ». Le premier objectif du PDU, « Reconquérir le centre-ville », est présenté comme étant articulé aux actions du «Projet de Centre-Ville»: « requalification des espaces publics et privés; développement de la fonction résidentielle par le retour des classes moyennes et des cadres supérieurs et l'arrivée de nombreux étudiants ; amélioration

\footnotetext{
${ }^{9}$ Les documents sélectionnés sont à la fois les plus cités par les différents acteurs politiques et techniques et les plus mobilisés dans les travaux analysant les politiques publiques dans le centre-ville de Marseille.

${ }^{10}$ Agence MCM, mairie de Marseille, Projet centre-ville (1999) Réhabilitation Marseille-centre, p. 10.
}

du cadre de vie ». Il est également souligné que l'objectif de revitalisation du centre-ville « qui cherche à attirer à nouveau des résidents et des consommateurs dont la pratique de l'automobile reste forte » devra être harmonisé avec des objectifs contradictoires « libérer l'espace publics des obstacles qui l'encombrent, au premier rang desquels les véhicules en stationnement » et la « limitation de l'usage de la voiture, au profit des modes de transport collectif et des modes légers (marche à pied, deux-roues) moins polluants ». Plus loin dans le document : «privilégier les modes collectifs et légers soulage l'espace public de la pression de l'automobile, facilitant la bonne gestion de l'espace qui redevient agréable à fréquenter, à parcourir, et attire au centre des consommateurs, des visiteurs et des résidents ». Globalement cette partie du PDU met en évidence que l'enjeu des actions proposées n'est pas tant l'accroissement de la pratique de la marche, déjà jugée très importante $(62 \%$ des déplacements internes au centre-ville) ${ }^{11}$, mais plutôt la lutte contre l'envahissement de l'espace public par l'automobile (en multipliant les zones piétonnes, semi-piétonnes ou apaisées).

Par ailleurs, le PDU adopté en novembre 2000 contient, en creux, une critique des actions antérieures menées sur le centre-ville en appelant dorénavant à une action globale et d'ampleur en faveur des modes doux ${ }^{12}$. En effet, les projets de piétonisation à Marseille ont souvent été limités dans leur périmètre et ont eu peu de retombées significatives sur la redynamisation commerciale $[19,20]$.

Plus récemment, l'Opération grand centre-ville vise à atteindre une attractivité renforcée du secteur « autour $\mathrm{du}$ commerce, de l'offre universitaire du savoir et du tourisme ». Parmi les différents objectifs déclinés quant à l'amélioration du fonctionnement urbain (la requalification des espaces publics, l'extension de l'offre résidentielle, le développement touristique), figure celui de «Restaurer la chalandise dans un centre-ville apaisé, animé et convivial $»^{13}$. La relance de l'activité commerciale va de pair avec une ambiance largement liée aux interventions urbanistiques et à l'identité renouvelée des espaces publics.

\footnotetext{
${ }^{11}$ Pour étayer ce propos : dans le même PDU, la partie du consacrée à l'action « Favoriser les modes de déplacement non polluants : vélo et marche » souligne que la marche est une pratique qui se déploie dans le centre-ville et les noyaux villageois et qu'il s'agit de favoriser son développement en périphérie où elle peut, pour certains déplacements de proximité, se substituer à la voiture. Ville de Marseille (2000) Plan de déplacements urbains, p. 86 .

12 « Il ne s'agit pas de se limiter à créer de mini-zones piétonnes, quelques rues éparses, en îlots autour de voies de grandes circulations car cela n'aurait pas d'effet sur la structure des déplacements, ni sur l'aménagement du centre et sa revitalisation (on créerait de petites zones commerçantes dynamiques le jour mais désertées la nuit et qui affaibliraient en outre les autres commerces) » Ville de Marseille (2000) Plan de déplacements urbains, p. 59.

${ }^{13} \mathrm{http}: / / w w w . v i e u x p o r t d e m a r s e i l l e . f r / i m a g e s / C h a p i t r e-3 . p d f$
} 
La définition de 15 axes de ravalement de façades (dont le Cours Belsunce) concerne en réalité des segments urbains fortement portés vers l'activité commerciale. En améliorant le bâti, en contribuant à sa mise en scène, les opérateurs envisagent ainsi de créer un décor favorable à la déambulation - loin des paysages aujourd'hui disgracieux dans lesquels alternent bâtiments abandonnés, dégradés puis subitement entretenus.

\section{La marche comme un flux et non comme un mode de vie}

\section{Les carences de l'espace public pour le piéton, un constat partagé}

Le diagnostic du PDU (2000) est sans appel concernant la place accordée à la marche à pied et au vélo dans la ville : « Le plus souvent les trottoirs sont étroits, voire très étroits, ils sont occupés par la voiture qui a tendance à envahir tout l'espace [...]. Bref, le piéton s'est vu progressivement confisquer ses trottoirs et contraints de slalomer autour de multiples obstacles : il a été marginalisé. Il n'existe pas véritablement d'itinéraire piéton continu et lisible permettant de rejoindre les pôles d'animation du centre-ville ».

La majorité des études commanditées sur le centreville de Marseille constate la présence d'espaces publics dégradés, principalement occupés par la voiture et en nombre insuffisant, notamment au niveau des places. Le SDEP, quant à lui, se réfère à l'histoire du centre pour expliquer « que les espaces publics actuels relèvent d'un urbanisme ancien, qui ne connaissait ni la voiture, ni les mobilités de centre à banlieue : à titre d'exemple, plus de $45 \mathrm{~km}$ de voies du centre-ville, soit $55 \%$, ont une largeur entre façades inférieure à 10 mètres, ce qui rend évidemment difficile la cohabitation des véhicules en circulation, des véhicules en stationnement et des piétons ». Ce document présente un espace public peu confortable pour le piéton (voies étroites, trottoirs réduits), souvent encombré de véhicules (et parfois même de conteneurs) et d'éléments de mobilier urbain (potelets, kiosques, panneaux indicateurs) - constituant autant d'obstacles -, et caractérisé par un manque de continuité dans les itinéraires, une absence de zones piétonnières en quantité et en qualité suffisantes... Le cheminement à pied dans le centre-ville apparaît comme « une épreuve » renforcée par la difficulté à traverser les axes de transit occupés par des véhicules aux « comportements particulièrement dangereux ». Le SDEP du centre de Marseille part ainsi du postulat que « l'espace public, longtemps resté le parent pauvre des politiques communales, doit faire l'objet de démarches ambitieuses et déterminées pour jouer à nouveau son rôle social et économique, dans une stratégie de reconquête positive passant par une libération progressive des usages indésirables, une requalification durable et une gestion rigoureuse ». Sur un périmètre d'une surface totale de 376 hectares (faisant l'objet de 680000 déplacements par jour) dont 116,5 ha en rues, places et surfaces assimilées, plus des deux tiers sont entièrement consacrés à l'automobile. Face à ce constat, le PDU, tout comme le SDEP, se donnent, entre autres ambitions, de restituer une partie de l'espace aux piétons.

La question du piéton dans la ville semble dès lors posée comme un simple problème d'espace nécessaire à une pratique circulatoire de la marche. Le piéton doit pouvoir disposer d'un espace suffisamment large, sans obstacle et continu. Cette approche perpétue la prise en compte du piéton en termes de flux et de largeur de trottoir.

\section{Une focalisation de la Ville sur les populations actives et consommatrices au détriment des populations résidentes}

Les typologies d'usagers suggérées dans les différents documents lorsqu'il est fait référence aux aménagements à destination du ou des piétons confirment bien la volonté de la Ville d'investir dans le centre pour une population ciblée attendue, extérieure au quartier.

Le Projet centre-ville ambitionne dès 1995 de « Faire revenir la population au cœur de la cité » et annonce des efforts engagés avec pour objectif de "séduire de nouveau les trois catégories de population : les résidents, afin d'attirer une population nouvelle (classes moyennes) ; les semi-résidents constitués essentiellement par une population étudiante et touristique ; les non-résidents, c'est-à-dire la population qui vient chaque jour en centre-ville pour travailler, consommer, se distraire ou effectuer des formalités administratives ». Les travaux de requalification annoncés comme des restructurations lourdes (sous l'égide d'un opérateur unique la société d'économie mixte Marseille Aménagement) sont destinés à dynamiser de façon significative le marché de l'immobilier et à rendre plus attractif le centre-ville. La « rénovation » des espaces publics (plus de $100000 \mathrm{~m}^{2}$ réalisés entre 1995 et 2001) est envisagée à la fois pour améliorer le cadre de vie, mettre en valeur le patrimoine architectural et urbain, et contribuer au développement économique. Dans ces documents, pour tous les travaux réalisés et envisagés, la municipalité affiche le parti pris de la priorité aux piétons en choisissant de rendre les voies soit piétonnes (interdiction aux véhicules sauf dérogations) soit semi-piétonnes (circulation des véhicules encadrée par des obstacles, vastes espaces pour les piétons). Les actions concernant les résidents figurent à la marge dans un paragraphe intitulé « Améliorer la vie quotidienne ». 
En effet, à l'exception de la mise en place d'un service Opération Allô Mairie (destiné à répondre aux problèmes urgents des Marseillais dans les domaines de la propreté, de la voirie et la sécurité), la majorité des actions programmées visent l'amélioration des conditions de fréquentation des citadins pratiquant le centre et notamment les consommateurs. Plus précisément sont envisagés la création de places de stationnement, le développement des transports en commun, le renforcement de la propreté (nettoyage des tags, de l'affichage sauvage, enlèvement d'objets encombrant, campagne de sensibilisation...) et enfin la sécurité (sécurité publique, gestion urbaine et services publics, lutte contre la toxicomanie, lutte contre les violences urbaines, prévention de la délinquance). Ces attentions sont considérées comme autant d'actions nécessaires à la pratique du centre-ville par des piétons attirés notamment par la fonction commerciale du secteur. Cette restructuration du centre s'accompagne en réalité de l'objectif de renforcer l'hypercentre commercial. Sont ainsi envisagés la création de liens entre les grandes artères commerciales et le Centre-Bourse, l'amélioration de l'environnement du consommateur, l'arrivée de nouvelles enseignes attirées par les facilités d'implantation et enfin la création d'une identité commerciale du centre-ville.

Après avoir mis en évidence la volonté de valoriser la fonction commerçante du centre-ville, nous allons maintenant regarder les identités d'usagers présentes dans ces documents.

Dans le PDU (2000), il est souvent fait cas des consommateurs et des résidents espérés, alors que les résidents actuels sont peu évoqués en tant que tels : ils sont englobés dans la catégorie de « ceux qui fréquentent » ou parcourent le centre-ville. Dans ce même document, lorsqu'il s'agit de recomposer l'offre de stationnement, le stationnement de surface semble être réservé aux visiteurs et aux consommateurs alors que les résidents sont invités à utiliser des parkings en ouvrage, le plus souvent souterrains.

Dans le SDEP quatre types d'usagers sont identifiés : « le résidant », « l'actif ou le visiteur d'activités », « le touriste et le visiteur d'agglomération ». Il est précisé qu'au regard du faible poids démographique du centre-ville (100 000 habitants) et du nombre de logements vacants, « Il est capital de reconquérir une clientèle résidentielle en centre-ville de Marseille ; le pari est donc de lui offrir une variété de produits immobiliers de qualité, mais aussi un cadre urbain d'excellente qualité ». Aucune mention n'est faite sur le profil socio-économique de l'habitant en place, en revanche la reconquête d'une clientèle résidentielle est clairement posée.

Dans l'Opération grand centre-ville, le piéton est pour l'instant un « objet non identifié »- bien que dans le texte une préoccupation générale d'accueil des étudiants et des touristes dans le centre-ville soit signalée. La réalisation des programmes en cours permettra à terme de comprendre à quel(s) public(s) s'adresse véritablement les aménagements.

\section{Absence d'appui sur les pratiques existantes}

Les documents consultés, qui s'intéressent à la situation de l'hypercentre de Marseille, parlent peu de l'appropriation des espaces publics par différents groupes sociaux. En premier lieu, les commerçants occupent les trottoirs soit au titre d'annexe de leur boutique, accroissant ainsi leur espace de vente, soit comme espace de stockage des cartons vidés de leur marchandise, voire de leurs déchets divers. Dans une autre catégorie, des vendeurs à la sauvette, originaires notamment du continent africain et de Chine, s'installent ponctuellement sur les trottoirs ou dans les espaces piétonnisés pour vendre leurs produits à même le sol, repliant le tout dans de grands morceaux de tissus pour s'enfuir au plus vite à l'arrivée de la Police. L'activité commerciale a donc tendance à prendre le dessus et à empêcher une circulation sereine des piétons contraints de descendre sur la chaussée pour continuer leur parcours dans la ville. Par ailleurs, dans des secteurs où les logements sont particulièrement exigus et les hôtels meublés nombreux, une population composée principalement d'hommes vient s'installer dans les espaces publics dès que le temps le permet profitant ainsi d'un espace de vie en plein air envisagé dans la continuité de l'espace privé peu confortable. L'absence de bancs est immédiatement palliée par l'installation de chaises d'intérieur ou de petits tabourets empêchant là aussi le passant de circuler le long des bâtiments.

Seul le SDEP aborde la question des pratiques tant du côté des usagers que des animateurs de la gestion urbaine et pose volontairement un constat sévère pour montrer « à quel point la démarche de reconquête sera longue et difficile ; car, aujourd'hui, l'espace public s'est banalisé, au sens où il assure, indistinctement et au mépris de toute logique ou de considérations de qualité, toutes les fonctions utiles : lieu de stationnement, de circulation, d'entreposage des déchets, d'occupations temporaires... Il en résulte un sentiment d'appropriation (légitime au demeurant : l'espace public appartient bien, comme son nom l'indique, à tous ceux qui composent la collectivité) qui se transforme très vite en utilisation abusive pour motifs individuels nuisibles à l'ensemble de la collectivité ».

Il y est question de "l'ingéniosité publique comme privée » déployée pour « s'approprier des espaces à l'évidence non conçus pour cela » (exemple de plots enlevés pour permettre le stationnement d'un véhicule entre un mur de façade et la rue) mais aussi d'une gestion qui témoigne de ce laisser-aller : «pauvreté générale des revêtements de surface le plus souvent marqués par des ouvertures 
de tranchées successives et rapprochées, pusillanimité dans l'affectation des espaces de voirie au détriment de l'efficacité du système de transport public, incohérence de la politique de tarification du stationnement, insuffisante répression des comportements illicites ».

\section{Une approche du piéton par les flux, plutôt que par les pratiques}

L'examen des documents d'urbanisme retenus révèle une approche de la marche essentiellement quantitative. Deux raisons peuvent expliquer ce penchant. En premier lieu les injonctions législatives en matière de déplacements appellent les communes à accroître la part modale de la marche et des modes doux en général. Une évaluation des PDU, demandée par le législateur et portant sur des résultats obtenus, nécessite la production de données chiffrées. En second lieu, l'objectif de redynamisation commerciale, développé dans la partie précédente a pour corollaire l'accroissement de la fréquentation du centre.

Aussi, une des préoccupations de la Ville est de savoir si la limitation de l'accès automobile au centre-ville est susceptible d'en pénaliser l'attractivité commerciale. Elle a donc commandité une étude portant sur « le comportement de la clientèle sur le tracé du tramway ». Portant sur la période 2004-2007, elle révèle que « La fréquentation piétonne constatée aux abords des stations de tramway met en exergue une légère augmentation des flux piétons ». Ce constat positif est rappelé dans le Bilan LOTI du tramway ${ }^{14}$, dont une partie traite des effets du rééquilibrage des espaces publics sur l'accroissement de flux piétons.

Dans les documents analysés, il est rarement fait référence à la promenade. Lorsqu'elle est évoquée, elle est associée à des espaces touristiques, mettant en connexion les composantes urbaines et paysagères du patrimoine historique de la ville. De manière générale, dans les PDU marseillais analysés, la marche est abordée sous un seul angle : celui de la promotion des modes « doux » ou « légers », appréhendée en termes de « circulation piétonne » (en opposition à la « circulation automobile » qui doit être réduite). Un seul projet, celui d'un réseau vert, permet d'aborder une autre dimension de la marche, le plaisir de la balade : "Mais plus qu'un mode de transport, la marche peut être un plaisir voire même un droit, qui tend à disparaître dans les villes tant l'espace réservé au piéton s'amenuise ». Reliant les principaux équipements générateurs de déplacements, les arrêts des transports collectifs et les parcs et jardins publics ce projet de réseau vert est présenté comme « réunissant l'utile et l'agréable » et

\footnotetext{
${ }^{14}$ AGAM et MPM (2008) Bilan LOTI Tramway. Établissement de l'état 0 .
}

pouvant être un lieu de balade. Ce projet, d'une autre teneur, a été assez rapidement abandonné.

\section{L'aménagement des espaces pour le piéton associé à la valorisation des espaces publics de prestige}

Le contenu des documents consultés, avant de s'intéresser $\mathrm{au}(\mathrm{x})$ piéton(s), distingue au moins deux grands types d'espaces publics : ceux liés au statut de métropole (espaces de prestige) et ceux animant la vie quotidienne de quartiers (espaces de proximité).

Dans le SDEP, les espaces publics sont regroupés en trois types : espaces exceptionnels (à l'échelle du centre-ville dont le Cours Belsunce - mais aussi à l'échelle du quartier), espaces courants, espaces des parcs et jardins.

Dans les documents relatifs à l'Opération grand centreville, les espaces publics sont annoncés comme «la pièce maîtresse d'un cœur de ville renouvelé ». Dans la perspective d'envisager ces espaces comme « signature du centre-ville », une attention particulière est accordée à la semi-piétonisation du Vieux-Port (qui a fait l'objet d'un concours international remporté par Norman Foster et Michel Desvigne), mais aussi à la « rénovation complète des places emblématiques »du centre. Sont également programmés la mise « en réseau des espaces publics pour en améliorer l'usage par les piétons » et la poursuite des aménagements piétonniers et semi-piétonniers existants. Il est annoncé que les espaces publics doivent (re)devenir des sites d'événements mais aussi des lieux adaptés aux services de proximité et à la vie quotidienne pour « servir de support à un nouveau maillage des déplacements doux grâce à la réalisation de pistes dédiées aux piétons et aux vélos et enfin de créer des espaces de verdure, nécessaires à l'harmonie du centre-ville ». Pour autant, les documents opérationnels mettent l'accent sur les espaces publics emblématiques médiatisés dans le cadre des manifestations de Marseille-Provence 2013 Capitale européenne de la culture, et minimisent l'intervention sur les autres espaces publics. Le type d'intervention sur les espaces publics dédiés à la vie quotidienne reste en attente du lancement des programmations envisagées sur les 35 pôles urbains du périmètre élargi du Grand centre-Ville.

Ces multiples résultats confirment que la Ville recherche, à travers ses aménagements programmés, ce que nous nommons « une marche dynamique », c'est-à-dire une marche utile, correspondant à un circuit d'activités précises (démarches administratives, fréquentation d'un équipement, accès au lieu de travail...), dans des temps contraints et qui laisse peu de place à flânerie. La «marche dynamique » fait écho au dynamisme recherché par une ville qui se projette comme métropole attractive. La marche dynamique renvoie à l'idée d'une « ville qui bouge », d'une « ville qui avance », 
d'une ville dans le mouvement. Cette marche est pratiquée par les populations actives (à Marseille dans un contexte de développement du secteur tertiaire les cadres remplacent les ouvriers, l'attaché-case devenant le symbole du renouveau économique), par les touristes... Les aménagements des espaces publics et la valorisation de certains itinéraires répondant à « un impératif de visibilité commerciale et urbanistique » visent à canaliser les flux sur une diagonale reliant la gare Saint-Charles au Vieux-Port [21]. C'est bien d'une « marche guidée » qu'il s'agit, bien loin de la flânerie, de la «marche libre », de la promenade ou encore de la circulation éparpillée de la population résidente dans son quartier de vie. La marche ponctuée de pauses reste le parent pauvre des programmations et les aménagements qui lui seraient nécessaires et notamment la présence de bancs, ne sont pas envisagés (ou de façon marginale).

\section{De promenade à espace de transit : le Cours Belsunce}

La démonstration, jusqu'ici étayée par l'examen des diagnostics et programmations contenus dans les documents d'urbanisme, est complétée par une étude de terrain : le Cours Belsunce constitue une illustration pertinente de notre propos. D'une part, cet espace est appréhendé dans les différents documents d'urbanisme comme un espace de prestige et à valoriser pour son caractère exceptionnel (présence d'architectures remarquables et d'équipements à l'échelle métropolitaine). D'autre part, le Cours est pratiqué par la population des quartiers populaires adjacents comme un espace du quotidien.

\section{Le « cours », figure urbaine de la promenade}

Le mot " cours », issu du latin cursus, forme dérivée du verbe currere, " courir », est utilisé pour dénommer des voies urbaines destinées à la promenade. Absent des dictionnaires d'urbanisme [22], il est défini par ceux de l'art des jardins comme "vaste enclos où une avenue plantée, proche de la ville, est offerte à la promenade en carrosse et à cheval » [23] ou par les dictionnaires généralistes comme « une avenue longue et large servant de promenade».

La figure urbaine du « cours » est introduite en France (à Paris, en 1618) par volonté de la reine Maria de Médicis qui importe de Florence la pratique de se promener en carrosse et à cheval dans un espace planté et clôturé, réservé à la cours et à l'aristocratie (Promenade des Cascine, à Florence). Jusqu'à cette époque, la promenade se faisait à pied, dans les jardins privés. À Paris, un espace oblong, d'environ $1800 \mathrm{~m}$ sur $300 \mathrm{~m}$, qui prend le nom de Cours-de-la-Reine, est alors réalisé entre les jardins des Tuileries et les pieds de la colline de Chaillot. Il comporte quatre rangées d'arbres et il est constitué d'une allée centrale et de deux contre-allées ; au milieu, un rond-point en demi-lune permettait aux équipages de faire demi-tour. Le Cours-de-la-Reine était un lieu fermé, comme son modèle florentin : l'accès se faisait par deux grilles situées aux extrémités et surveillées par des portiers, qui n'autorisaient que les membres de l'aristocratie à rentrer. Cet espace est présenté en 1649 comme le « Grand Promenoir de Paris » et rentre dans les dictionnaires dès la fin du siècle $[24,25]$.

Le cours, en tant que figure urbaine, se répand ensuite dans d'autres villes françaises, notamment dans les régions au bord de la Méditerranée, où les conditions climatiques facilitent les pratiques sociales qui y sont associées [26]. À Marseille, le Cours Belsunce, ouvert en 1670, ne joue pas seulement le rôle de promenade aristocratique, mais aussi celui de jonction et de raccord morphologique entre la ville ancienne et les nouveaux quartiers de l'extension urbaine. Il permet également d'accéder à une des entrées plus importantes de la ville, la porte d'Aix.

\section{Retrouver les attributs d'un prestige passé : un enjeu pour le Cours Belsunce}

Dans l'histoire marseillaise, le Cours Belsunce fut, jusqu'au milieu du dix-neuvième siècle, un espace de représentation cher aux négociants et à l'aristocratie. En 1857 les travaux d'embellissement transforment la Canebière en un axe concurrent au Cours [27]. C'est sur cet axe que s'installeront les grands hôtels et les cafés, images de la ville bourgeoise. Ce nouvel espace sera privilégié et le Cours Belsunce perdra définitivement son prestige avec l'arrivée des omnibus qui relient la ville à ses noyaux villageois. Les transformations postérieures à la seconde guerre mondiale ont profondément modifié la forme du Cours Belsunce. Dans les années 1960, sa partie occidentale a fait l'objet d'un programme de rénovation urbaine visant la réalisation d'un centre d'affaires qui ne pourra être véritablement concrétisé : seuls seront construits trois tours de logements, une barre à destination tertiaire et un centre commercial, le Centre-Bourse. Le Cours perd les caractères qui le définissent (espace fermé et encadré par des architectures de qualité) : de pièce urbaine, il devient un espace de transit entre la Porte d'Aix (débouché de l'autoroute A7) et le Vieux-Port. Il est également un axe fort du dispositif commercial mis en place par les populations issues des différentes vagues migratoires accueillies dans le quartier Belsunce, renforçant ainsi son rôle de « carrefour » $[28,29,30]$.

Depuis 2000, le Cours Belsunce est le support de deux équipements majeurs de la ville, la bibliothèque municipale à vocation régionale de l'Alcazar (2004) et le tramway qui la dessert (2007). Ces derniers sont le signe d'une volonté 
de transformation des usages antérieurs et appellent la venue d'une population nouvelle. Cette transformation tente d'accélérer la requalification du quartier Belsunce entamée depuis plusieurs décennies (procédures de réhabilitation de type OPAH, PRI, RHI ; transformation d'une rue anciennement vouée à la prostitution en « Rue des Arts », création du Mémorial de la Marseillaise), mais aussi de ses accès (restructuration de la Porte d'Aix et de la gare Saint-Charles dans le cadre du projet Euroméditerranée).

Le Cours faisant l'objet d'une attention particulière en termes d'aménagement, son aspect physique a véritablement changé (architectures restaurées, espace public redistribué et requalifié, nouveaux équipements), mais ses activités et ses pratiques restent encore marquées par l'histoire du peuplement de ce cœur de ville et par la proximité de quartiers populaires résidentiels et commerciaux. Les locaux en rez-de-chaussée des immeubles de sa partie orientale sont occupés par des commerces dédiés à l'habillement et à la personne à des prix discount [31]. Cette activité est, en quelque sorte, complétée par la présence ponctuelle de vendeurs à la sauvette installés à même le sol sur le vaste trottoir du Cours. Mama africaines ou plus récemment vendeurs chinois visent le flot de passants, consommateurs potentiels, attirés par l'ensemble commercial du secteur [32].

\section{Un Cours aux allées déséquilibrées entre mouvement et immobilité}

Le Cours Belsunce se présente aujourd'hui comme un espace déstructuré, où les multiples transformations des deux rives ont répondu à des logiques d'aménagement distinctes et créé un déséquilibre en termes de composition urbaine. Seuls les alignements d'arbres rappellent l'ancien lieu de promenade. Le mouvement domine l'espace - traversé dans toutes les directions par des piétons et des véhicules. Les qualités de l'immobilité semblent oubliées : attendre, flâner, pique-niquer ou simplement regarder les gens passer sont des activités non-prévues par les concepteurs de l'espace urbain [33].

Dans une ville méditerranéenne où le climat est propice à une occupation prolongée des espaces publics, il est pertinent d'analyser les possibilités offertes en termes d'immobilité autrement dit les aménagements pensés à destination des promeneurs. Préciser que Marseille est une ville méditerranéenne n'est pas anodin, car au-delà du climat c'est une culture spécifique dans un rapport singulier aux espaces publics qui doit être interrogée. La pratique des rues-salons telles qu'elle s'affirme dans les quartiers populaires de Naples ou de Valence (Espagne)

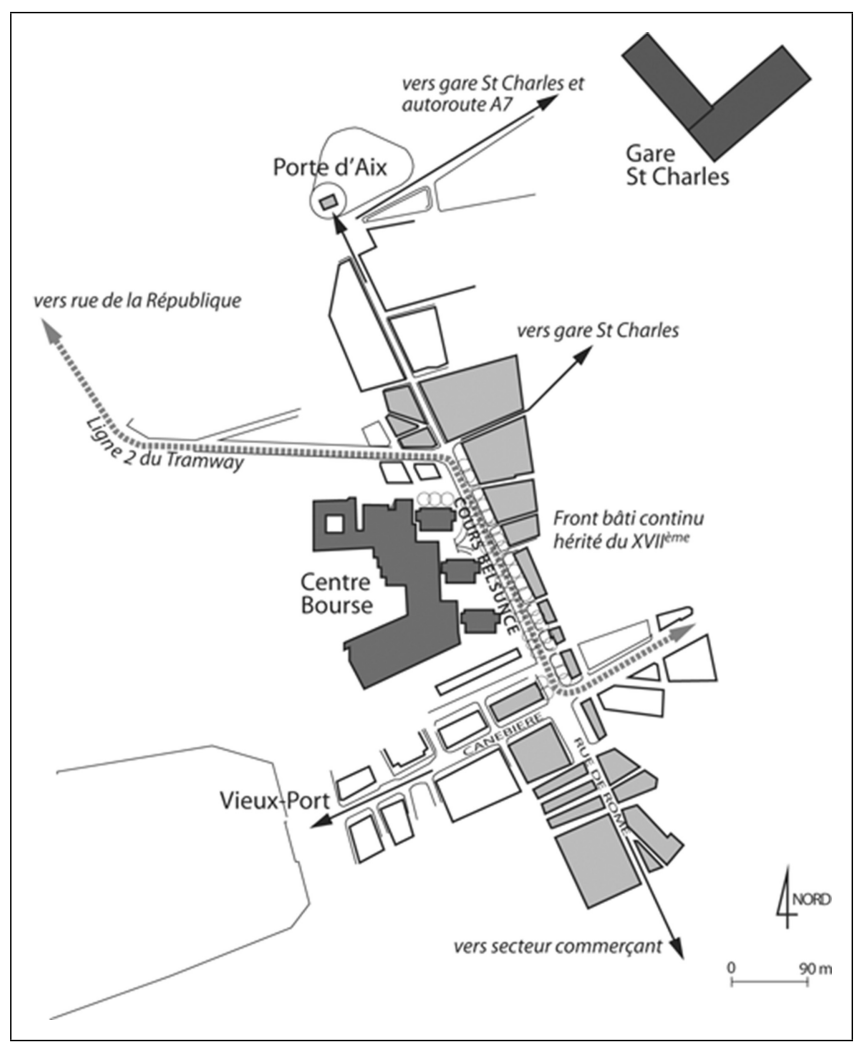

Fig. 1 Localisation du Cours Belsunce dans l'hypercentre de Marseille

Source : Frédérique Hernandez

est observable dans un certain nombre de quartiers de Marseille et notamment l'été à Belsunce quand les populations sortent chaises et tabourets sur les trottoirs pour y prendre le frais et échanger. Étonnamment à Marseille, il est difficile de trouver un banc pour s'assoir. Il est vrai que l'espace central a longtemps été totalement envahi par la voiture, ne donnant pas nécessairement l'envie de s'arrêter à hauteur des véhicules (vue, pollution, bruit...), et que de rares espaces de trottoirs élargis ou les places ont été occupés par des cafetiers. Il semblerait que le projet de transformation du centre s'adresse prioritairement aux nouvelles populations espérées au détriment des populations en place et de leurs attentes.

Sur un Cours à la croisée de multiples modes de transport (arrêt de tram, station de bus, métro) et encore fortement fréquenté par les automobiles, une circulation intense marque les ambiances et soulève la question de la place accordée $\mathrm{au}(\mathrm{x})$ piéton(s). C'est bien au pluriel que cet usager des espaces publics doit être appréhendé, ne serait-ce que pour marquer la double distinction entre le piéton en situation de " marche dynamique » et celui en «marche libre », mais aussi le piéton extérieur au quartier et le piéton-résident. 


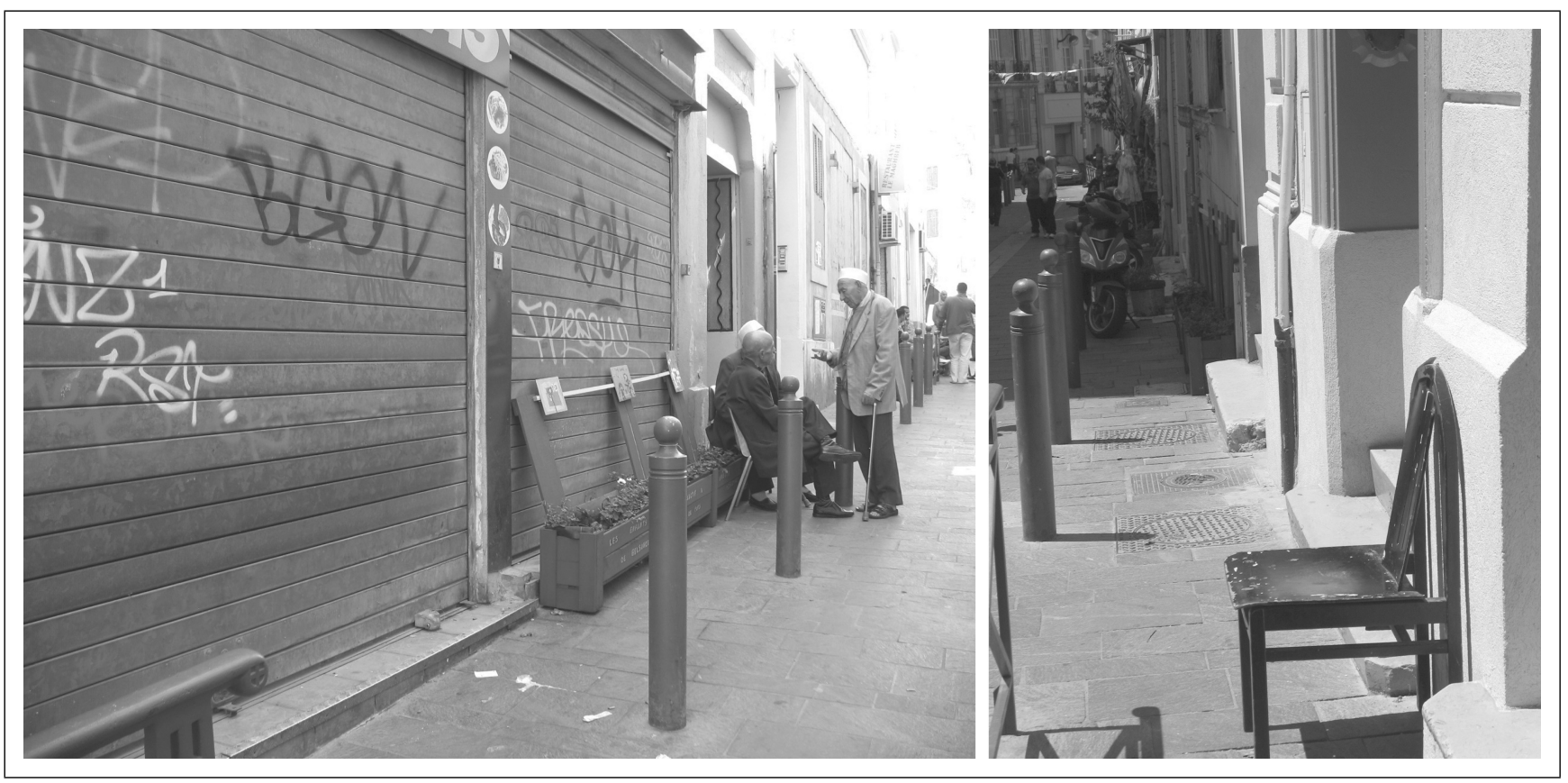

Fig. 2 Dans une rue débouchant sur le Cours Belsunce les résidents utilisent leurs propres chaises

Source : photos Brigitte Bertoncello

\section{Le piéton-résident oublié des aménagements du Cours}

La rareté d'espaces publics à l'intérieur du quartier Belsunce, caractérisé par une forte densité du bâti, amène les habitants à s'approprier le Cours qui émerge par contraste avec les rues du quartier comme un espace lumineux, arboré et animé. Il s'agit d'un espace de connexion avec le reste de l'hypercentre, en partie piétonnier, dans lequel les aménagements récents ont privilégié les fonctions de transit, de circulation et de desserte des parkings souterrains (plutôt que l'arrêt, la pause). L'absence de mobilier urbain empêche les piétons de s'attarder ou de s'installer librement sur cet espace - à l'exception des seules terrasses de cafés qui offrent une halte accompagnée d'une consommation. Ce choix des édiles exprime la volonté d'éviter l'installation de populations mal-venues dans la vitrine en construction ${ }^{15}$ que constitue l'hypercentre aujourd'hui ; il est en contradiction avec les tendances actuelles qui recherchent un bon équilibre entre transit et séjour dans les espaces publics urbains.

L'absence de mobilier pour le promeneur, d'agréments dans l'espace public incitant à la pause illustre le décalage

\footnotetext{
${ }^{15}$ Nouredine Abouakil, président de l'association Un centre-ville pour tous « qui défend le maintien de la diversité sociale dans le centre », déclare «Une partie de l'élite marseillaise préfère qu'il n'y ait pas d'espace public plutôt qu'il soit "mal occupé" ». Le Point (10 juillet 2010)
}

constaté entre la politique annoncée et la réalité de l'aménagement.

Face à l'insatisfaction générée par les programmations urbanistiques sur les espaces publics, des initiatives spontanées, assez inventives, sont impulsées par des individus (le plus souvent organisés en collectif) se mobilisant pour l'amélioration de leur cadre de vie. Il est sans doute ici possible de parler d'une population qui - en créant son propre aménagement - devient maîtrise d'œuvre. En 2009, une habitante du quartier Belsunce fait le constat d'une grande diversité sociale, culturelle et générationnelle des populations en présence sur le Cours Belsunce sans que la possibilité de s'assoir y soit proposée. Elle déclare alors : «Si vous allez voir dans les grandes villes européennes, un espace comme celui-ci serait un rambla comme à Barcelone, un espace où les gens discutent, où les gens, si ils veulent, peuvent manger un sandwich... C'est par essence un objet d'accueil, un objet qui nous dit la bienvenue dans cette ville sans être obligé de payer pour être assis si vous êtes fatigués, si vous êtes malade, tout simplement si vous voulez contempler la rue, vous pouvez le faire ${ }^{16}$. Comprenant, après avoir contacté la Ville sur le sujet, que «c'était une politique de la ville de ne pas mettre de bancs pour qu'il n'y ait pas de désordre dans la ville » elle se rapproche de l'association El otrolugar/L'autre lieu pour

\footnotetext{
${ }^{16}$ Interview de Maritza Fuentes, 7 juin 2009, WebTV réseau marseillais.
} 


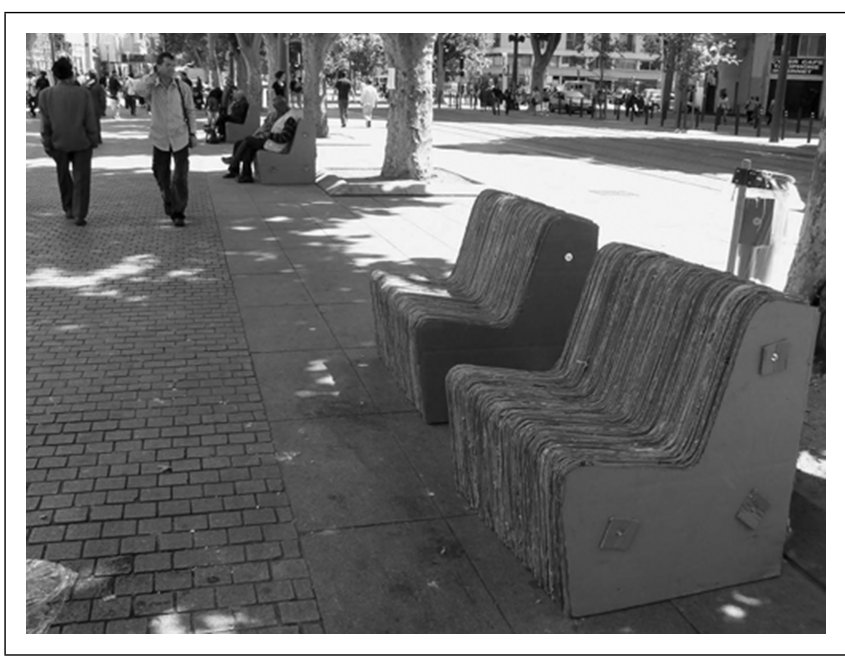

Fig. 3 Sur le Cours: installation temporaire de bancs par l'association El otrolugar/L'autre lieu

Source : http://ciudadesimaginables.blogspot.fr/2009/05/lesbancs-publics-en-carton-sont-la.html

produire des bancs à partir de cartons récupérés dans les différents quartiers commerçants du centre. Installés sur le Cours Belsunce durant une semaine, ces bancs seront pleinement appropriés tant par les piétons-résidents que par les piétons à la recherche d'une halte entre deux achats. L'opération n'a pas généré comme il l'avait été imaginé, une réaction constructive de la Ville et l'aménagement du Cours n'en a pas été modifié pour autant. Aujourd'hui, les piétons-résidents du quartier Belsunce désireux de «se poser » sur le Cours Belsunce, oscillent entre s'appuyer sur les plots de l'espace public, arriver avec leur pliant, ou encore négocier une chaise avec les cafetiers quand les terrasses ne sont pas trop occupées.

\section{Identité plurielle du piéton du Cours Belsunce}

Les documents d'urbanisme préoccupés par le sort des espaces publics, nous l'avons vu, ne donnent pas une image complète de l'identité et des intentions du piéton. Différents temps d'observation sur le Cours Belsunce ont permis de dégager des éléments qui pourraient à terme faire l'objet d'une typologie. Ainsi il convient de distinguer, dans un premier temps, la population résidant sur place et se déplaçant à pieds depuis les rues adjacentes, la population arrivée par les transports en commun (la marche étant le complément premier des déplacements en transport collectif) et les automobilistes sortant des parkings et rejoignant le centre-ville. Bien que l'observation permette de distinguer les touristes des actifs rejoignant leur lieu de travail ou encore des citadins en shopping, un questionnaire serait nécessaire pour préciser leurs motivations à marcher sur le Cours Belsunce. Dans un deuxième temps, il est possible de retrouver le piéton en marche active emporté par les flux de passants de celui qui s'inscrit dans l'immobilité, autrement dit distinguer le «piéton-consommateur » du « piéton-flâneur».

Selon le type de piéton, sa catégorie socio-professionnelle, son rapport à la marche (choisi ou subi), les attendus concernant les aménagements et les ambiances différent.

$\mathrm{Si}$, dans d'autres agglomérations, s'affiche en centre-ville le piéton qui marche par engagement citoyen et qui porte une attention particulière à son environnement urbain (esthétique, ambiance), à Marseille, et sur le Cours Belsunce, on trouve principalement le piéton qui marche par contrainte et par nécessité se préoccupant peu des ambiances mais attentif à la sécurité routière et publique (agressions éventuelles). Les travaux de Sylvie Miaux distinguant « la marche comme engagement dans un mode de vie », «la marche utilitaire vécue comme une nécessité », et enfin " la marche vécue comme une contrainte », pourraient être réinterrogés au prisme de la situation marseillaise. Sur le Cours Belsunce, les piétons-résidents appartiennent plutôt aux deux dernières catégories.

En définitive le Cours Belsunce ne présente ni les qualités de sécurité attendues (mauvaise lisibilité des voies à emprunter pour le piéton et mise en tension fréquente entre tram/piéton et automobile/piéton) ni les aménagements nécessaires aux piétons désireux de s'inscrire dans l'immobilité. La politique à l'œuvre à Marseille en matière de requalification des espaces publics semble avoir oublié le piéton-résident et reste caractérisée par des aménagements standardisés avec une faible prise en compte de l'identité méditerranéenne (des lieux et des populations) et des pratiques qui y sont associées. Prendre en compte cette spécificité renforcerait l'image populaire de la ville, une dynamique contradictoire avec le souhait clairement affiché d'attirer de nouvelles populations à partir notamment d'espaces-vitrine. La faible prise en compte de ces promeneurs pourrait être liée au fait qu'ils sont pauvres et ne correspondent pas aux populations attendues en centre-ville.

\section{Conclusion}

Les aménagements de l'espace public réalisés ces dix dernières années à Marseille s'inscrivent dans une dynamique globale de changement d'image destiné à rendre le centre attractif. C'est ce que révèle l'étude des documents produits depuis le milieu des années 1990, qu'ils s'agissent de diagnostics techniques ou de documents stratégiques. Le document intitulé Projet centre-ville souligne la nécessité de « faire revenir plus de consommateurs » et «d'améliorer 
l'environnement du consommateur ». Les Plans de déplacements urbains successifs mettent une partie de leur contenu au service de l'objectif de reconquête du centre-ville. La redynamisation du centre semble avant tout d'ordre économique ou tournée vers l'accueil d'une population nouvelle, classes moyennes et supérieures, étudiants ${ }^{17}$.

Le confort de l'espace public et le développement de la marche reviennent de façon récurrente dans les documents. À l'instar d'autres agglomérations, pour les édiles marseillais le piéton devient un des principaux destinataires de la promotion urbaine. Mais de quel piéton s'agit-il ? Toujours à travers l'examen des documents produits, nous avons cherché à saisir la ou les manière(s) dont étaient envisagés le piéton, ses pratiques et la façon dont se concevaient les aménagements nécessaires. Les typologies d'usagers suggérées, lorsqu'il est fait référence aux aménagements à destination du ou des piétons, confirment la volonté d'investir dans le centre pour une population ciblée, attendue, extérieure au quartier. C'est une « marche dynamique » qui est favorisée, une marche qui renvoie à l'image d'une « ville qui bouge », d'une ville dans le mouvement.

L'analyse des documents révèle également le peu d'appui sur les pratiques existantes et la faible proportion des références aux populations résidentes. Or, celles-ci sont amenées, du fait de leur fragilité économique, à se déplacer majoritairement à pied. Nous nous sommes alors demandés si les aménagements en faveur du piéton réalisés ou en cours à Marseille répondaient aux besoins de cette mobilité contrainte. Pour cela, nous avons étudié un espace public de l'hypercentre populaire : le Cours Belsunce. Il est aujourd'hui, dans le même temps, un lieu de prestige à valoriser par son caractère patrimonial et un espace du quotidien pratiqué par la population des quartiers adjacents. Les aménagements récents y ont privilégié les fonctions de transit et de circulation, plutôt que l'arrêt ou la pause. La « marche contemplative » semble délicate sur le Cours Belsunce, alors même que cet espace est une figure de la composition urbaine initialement dédiée à la promenade. L'absence de mobilier urbain empêche les piétons de s'attarder. Les résidents des quartiers alentours expriment leur insatisfaction et leur difficulté à pouvoir se poser sur le Cours. Le promeneur et le piéton-résident semblent avoir été oubliés dans la politique de requalification à l'œuvre à Marseille.

Dans son ouvrage Pour des villes à échelle humaine, Jan Gehl appelle les municipalités à remettre l'humain au centre des préoccupations de l'urbanisme [34]. Sa lecture sensible et sensorielle de la pratique de la marche appréhende le

\footnotetext{
${ }^{17}$ Cette démonstration pour gagner en intensité pourrait être confortée par une analyse d'autres espaces publics de l'hypercentre de Marseille et complétée par des enquêtes auprès de leurs usagers.
}

piéton comme être universel et remet en question l'approche par catégories d'usagers, de fait sélective. En creux, ces propos interrogent la capacité des édiles à favoriser ou non, à travers leur choix d'aménagement, le vivre ensemble.

\section{Références}

1. Dell'Umbria A (2009) Une histoire passionnelle de Marseille. $Z$ Revue itinérante d'enquête et critique sociale 2: 40-49.

2. Hernandez F (resp.), Bertoncello B, Méjean Ph, Bertoni A (2013) Marseille: Les fragilités comme moteurs pour l'invention d'une centralité métropolitaine originale? Rapport de recherche au PUCA, GIP AIGP, 26 Juillet 2013, 206 p.

3. Peraldi M (2001) La métropole déchue (Belsunce breakdown). In: Donzel A (ed.) Métropolisation, gouvernance et citoyenneté dans la région urbaine marseillaise Maisonneuve et Larose, Paris, pp.

4. Bertoncello B (2010) Euroméditerranée à Marseille: renouveler la ville populaire ? In: Cailly L, Vanier M (eds.) La France, une géographie urbaine. Armand Colin, Paris, pp. 184-189.

5. Donzel A. (1998) Marseille, l'expérience de la cité. Anthropos/Economica, coll. « Villes », Paris, 196 p.

6. Cullen G (1961) Townscape. Architectural Press, London, 315 p.

7. Lynch K (1960) The Image of the City. MIT Press, Cambridge/London, $194 \mathrm{p}$.

8. http://www.cnu.org/charter

9. Ascher F (2004) Le sens du mouvement : modernités et mobilités. In: Allemand S, Ascher F, Lévy J (eds.) Le sens du mouvement. Modernité et mobilités dans les sociétés urbaines contemporaines. Éditions Belin, Paris, pp 21-34.

10. Lavadinho S (2009) The multimodal walker: potentials for combining walking and public transport at the agglomeration scale. In: STRC Conference Proceedings, Ascona.

11. Lavadinho S (2010) Evaluating the potential for intercity travel combining public transport and active modes. IAMF, Geneva.

12. Turcot L (2007) Le promeneur à Paris au XVIII ${ }^{\mathrm{e}}$ siècle. Gallimard, Paris, $440 \mathrm{p}$.

13. Lavadinho S (2011) Le renouveau de la marche urbaine. Terrains, acteurs et politiques. Thèse de doctorat en géographie - ENS Lyon, $681 \mathrm{p}$.

14. Chaudoir P (2009) La rue : une fabrique contemporaine de l'imaginaire urbain. In: Rautenberg $\mathrm{M}$ L'imaginaire de la ville, le regard et le pas du citadin. Actes Sud, Arles, pp 51-64.

15. Lavadinho S, Lévy J (2010) Marcher avec les transports et la ville. Les rapports prospectifs-RATP, Paris.

16. Lavadinho $\mathrm{S}$ (2008) Chemins de traverse et lignes de désir. Urbanisme 359: 66-68.

17. Winkin Y, Lavadinho S (eds.) (2008) Des villes qui marchent, tendances durables en urbanisme, mobilité et santé. Projet ANR SEST 05 019, Université de Lyon, ENS-LSH.

18. Miaux S (2008) Comment la façon d'envisager la marche conditionne la perception de l'environnement urbain et le choix des itinéraires piétonniers. L'expérience de la marche dans deux quartiers de Montréal. Recherche Transports Sécurité 101: $327-352$.

19. Vaudur N (1982) Les espaces piétonniers en Provence-Côte d'Azur. Annales de Géographie 506: 442-453.

20. Norbert F (2003) Piétonisation et relance de l'activité commerciale dans le centre-ville de Marseille. Méditerranée 100: 5-10.

21. Vaudour N (2002) La mobilité et la ville: l'exemple de l'agglomération marseillaise. Méditerranée 98: 83-90.

22. Choay F, Merlin P (2009) Dictionnaire de l'urbanisme et de l'aménagement. Puf, Paris, 961 p. 
23. Conan M (1997) Dictionnaire historique de l'art des jardins. Hazan, Paris, $464 \mathrm{p}$.

24. Langlois D (1649) Le Cours de la Reyne ou le Grand Promenoir des Parisiens, Paris, $16 \mathrm{p}$.

25. Furetière A (1690) Dictionnaire universel : contenant généralement tous les mots françois tant vieux que modernes, et les termes de toutes les sciences et des arts. Arnout \& Reinier Leers, Paris, 3 vol.

26. Pinson D (1996) Fès et Aix, contact et spectacle ou les conditions culturelles de la forme et de la pratique des espaces publics. In: L'espace public dans la ville méditerranéenne. Éditions de l'Espérou, Montpellier, pp 231-245.

27. Roncayolo M (1996) Marseille : les territoires du temps. Éd. locales de France, Paris, 135 p.
28. Tarrius A (1998) Arabes de France dans l'économie mondiale souterraine. Éditions de l'Aube, La Tour d'Aigues, $219 \mathrm{p}$.

29. Temine E (2008) Marseille transit : les passagers de Belsunce. Autrement, Paris, $1369 \mathrm{p}$.

30. Bertoncello B, Bredeloup S (eds.) Marseille, carrefour d'Afrique. Hommes et Migrations 1224: 1-81.

31. Véronique Manry V (2006) Marseille Discount : sociabilités, échanges et mise en scène de l'abondance. P'tits Papiers Éditions, Marseille, 2006.

32. Bertoncello B, Bredeloup S (2004) Colporteurs africains à Marseille - un siècle d'aventure. Autrement, Paris, $166 \mathrm{p}$.

33. Wall E, Waterman T (2009) Urban Design. AVA Publishing, Worthing, $184 \mathrm{p}$.

34. Gehl J (2013) Pour des villes à échelle humaine. Les Éditions écosociété, Montréal, 273 p. 